\title{
The gas content of peculiar galaxies: Counterrotators and polar rings $\mathbf{s}^{\star, \star \star}$
}

\author{
D. Bettoni ${ }^{1}$, G. Galletta ${ }^{2}$, S. García-Burillo ${ }^{3}$, and A. Rodríguez-Franco ${ }^{4,5}$ \\ 1 Osservatorio Astronomico di Padova, Vicolo Osservatorio 5, 35122 Padova, Italy \\ 2 Dipartimento di Astronomia, Università di Padova, Vicolo Osservatorio 2, 35122 Padova, Italy \\ 3 Observatorio Astronómico Nacional-OAN, Apartado 1143, 28800 Alcalá de Henares-Madrid, Spain \\ 4 Departamento de Matemática Aplicada (Biomatemática) Sección Departamental de Optica, \\ Universidad Complutense de Madrid, Av. Arcos de Jalón s/n, 28037 Madrid, Spain \\ 5 Nobeyama Radio Observatory, Nobeyama, Minamimaki, Minamisaku, Nagano 384-1305, Japan
}

Received 23 February 2001 / Accepted 7 May 2001

\begin{abstract}
This paper studies the global ISM content in a sample of 104 accreting galaxies, including counterrotators and polar rings, which spans the entire Hubble sequence. The molecular, atomic and hot gas content of accretors is compared to a newly compiled sample of normal galaxies. We present results of a small survey of the $J=1-0$ line of ${ }^{12} \mathrm{CO}$ with the $15 \mathrm{~m}$ SEST telescope on a sample of 11 accretors (10 counterrotators and 1 polar ring). The SEST sample is enlarged with published data from 48 galaxies, for which observational evidence of counterrotation in the gas and/or the stars has been found. Furthermore, the available data on a sample of 46 polar ring galaxies has been compiled. In order to explore the existence of an evolutionary path linking the two families of accretors, the gas content of counterrotators and polar rings is compared. It was found that the normalized content of cold gas $\left(M_{\text {gas }} / L_{B}\right)$ in polar rings is $\sim 1$ order of magnitude higher than the reference value derived for normal galaxies. The inferred gas masses are sufficient to stabilize polar rings through self-gravity. In contrast, it was found that the cold gas content of counterrotators is close to normal for all galaxy types. Although counterrotators and polar rings probably share a common origin, the gas masses estimated here confirm that light gas rings accreted by future counterrotators may have evolved faster than the self-gravitating structures of polar rings. In this scenario, the transformation of atomic into molecular gas could be enhanced near the transition region between the prograde and the retrograde disks, especially in late-type accretors characterized by a high content of primordial gas. This is tentatively confirmed in this work: the measured $\mathrm{H}_{2} / \mathrm{HI}$ ratio seems larger in counterrotators than in normal or polar ring galaxies for types later than S0s.
\end{abstract}

Key words. galaxies: ISM - galaxies: interactions - galaxies: evolution - galaxies: peculiar - radio lines: galaxies - submillimeter

\section{Introduction}

The existence of kinematically decoupled disks of gas and/or stars with anti-parallel spins has been reported for a significant number of galaxies (see Galletta 1996, for a review). The phenomenon of counterrotation may be seen in the ionized gas (Bettoni 1984) but in almost half of the reported cases it is found in pure stellar disks (see Rubin 1994 for a review), being in some cases accompanied by gas counterrotation. Evidence of kinematical decoupling for the cold gas, either atomic or molecular, is also present

Send offprint requests to: G. Galletta,

e-mail: galletta@pd.astro.it

* Based on observations collected at SEST telescope, European Southern Observatory, La Silla, Chile.

** Table 1 is only available in electronic form at http://www .edpsciences.org in a high percentage of counterrotating galaxies (Bettoni et al. 1991; Braun et al. 1992; Casoli et al. 1998; van Driel \& Buta 1993, Sage \& Galletta 1994; García-Burillo et al. 1998, 2000).

Different scenarios have been proposed to explain the counterrotation present in elliptical and disk galaxies. Most of them invoke the capture of matter which comes from outside the acceptor galaxies. The various models examine different masses and time-scales involved in the accretion process. An external origin is also invoked to explain the existence of polar ring galaxies, where gaseous disks or rings are seen to rotate almost perpendicularly with respect to the main stellar body of the system (Whitmore et al. 1990). However, the link between polar rings and counterrotators remains unclear. Alternatively, it has been suggested that a primordial mechanism, 
invoking a dissipationless cosmological collapse perturbed by tidal fields, could explain the formation of counterrotating galaxies (Harsoula \& Voglis 1998).

Within the accretion scenario, the morphology of the acceptor system and its dynamic evolution would depend on several factors: the nature of the accreted matter (gas and/or stars), the ratio between the accreted mass and that of the acceptor galaxy and, finally, the accretion speed. Whereas the collision between equally massive galaxies may lead to a merging like the "Antennae", ending up as a giant elliptical galaxy with counterrotation (Hernquist \& Barnes 1991), the disruption of the acceptors disk could be avoided by progressive infall of gas whose spin is anticorrelated with the main stellar body (Quinn \& Binney 1992; Thakar \& Ryden 1996; Voglis et al. 1991). However, the accretion of a gas-rich satellite may heat the stellar disk (Thakar \& Ryden 1996). Observations show that lenticulars and spiral galaxies hosting counterrotation do not necessarily present disrupted stellar disks. Their stellar kinematics appear globally regular (Bettoni et al. 1991) and the stellar disks show hardly any sign of thickening (Rubin et al. 1992). The end product of the accretion process at the present epoch seems to have reached, in most of the known studied cases, an equilibrium configuration for the stellar component. Either the time-scales to reach equilibrium are short enough or, alternatively, accretion caused no traumatic changes in the kinematics of the stars.

The gas, however, is expected to reflect the consequences of the accretion process more violently than the stars. If gas was accreted by a disk galaxy with a non negligible amount of interstellar gas, a strong interaction between the accreted and the primary gas is likely. The existence of violent cloud-cloud collisions (the relative velocity between the interacting clouds would be: $v \sim 2 v_{\text {rot }}$ ) and the highly dissipative nature of the encounters might lead to the onset of large-scale shocks. These might convert the atomic gas into molecular gas (Braine \& Combes 1992; Sage \& Galletta 1993; Young \& Knezek 1989) and eventually induce starbursts (Wang et al. 1992; Read \& Ponman 1998; García-Burillo et al. 2000). If the described scenario holds, one would expect that the content of molecular gas would be higher in counterrotating galaxies than in a comparison sample of non-interacting galaxies of the same Hubble type. On the other hand, if the origin of counterrotation is primordial, or alternatively, if large-scale shocks are not efficient or short-lived, the $\mathrm{H}_{2}$ content should be similar for counterrotating and normal galaxies.

It is also unclear whether polar rings and counterrotators represent different steps in the process of mass accretion. A comparison of their $\mathrm{H}_{2}$ content could reveal if there is an evolutionary link between the two families of accretors.

This paper represents a first step in answering some of the above-mentioned questions by studying the global gas content in a sample of counterrotators. In this work we estimates the content of molecular, atomic and hot gas for a sample of 58 galaxies of different morphological Hubble types and of different types of counterrotation. Molecular gas masses are derived from ${ }^{12} \mathrm{CO}(J=1-0)$ observations made with the $15 \mathrm{~m}$ SEST radiotelescope on 10 galaxies with counterrotation and 1 polar ring (Sect. 2). Results from these new observations are described in Sect. 4 . The published data for 48 objects where there are indications of counterrotation in the gas and/or stars have been compiled and added to this sample (see Sect. 5). The $\mathrm{H}_{2}$ content of counterrotating galaxies will be studied relative to a comparison sample of normal galaxies that was built up from the literature, as described in Sect. 6. The gas content of counterrotators has also been compared with those of polar ring galaxies, using available data from the literature (see below for detailed references). A similar comparative study has been done with the HI content (from various sources), with the warm dust mass (derived from IRAS data) and with the amount of hot gas, the dominant gas component in early type objects (values derived from X-ray data taken by ROSAT and by EINSTEIN).

\section{The SEST sample}

The 11 galaxies in our sample observed using the SEST telescope were selected from the list of objects published by Galletta (1996). The galaxies are described individually in Sect. 4, together with the main results inferred from this CO study. The relevant parameters of the systems, such as the diameters $\left(D_{25}\right.$ : the de-projected linear diameter corresponding to the blue isophote at $25 \mathrm{mag} \operatorname{arcsec}^{-2}$ ), absolute $B$ magnitudes $\left(M_{B}\right)$, distances $(d)$ and morphological types are shown in Cols. 3-6 of Table 1 . These data have been extracted from the recently up-dated Lyon-Meudon database LEDA (Paturel et al. 1997).

The conversion factor between the integrated intensities of ${ }^{12} \mathrm{CO}(J=1-0)$ and the $\mathrm{H}_{2}$ column densities was taken from Strong et al. (1988), i.e.:

$\chi=N\left(\mathrm{H}_{2}\right) / I_{10}=2.3 \times 10^{20} \mathrm{~mol} / \mathrm{K} \mathrm{km} \mathrm{s}^{-1}$.

The total mass of molecular hydrogen (in $M_{\odot}$ ) under the $45^{\prime \prime}$ beam of SEST for the observed galaxies was obtained from $N\left(\mathrm{H}_{2}\right)$ using:

$M\left(\mathrm{H}_{2}\right)=7.8 \times 10^{-16} \times N\left(\mathrm{H}_{2}\right) \times d^{2}\left(M_{\odot}\right)$

where $d$ is the galaxy distance in $\mathrm{Mpc}$ and $N\left(\mathrm{H}_{2}\right)$ the measured column density in mol $\mathrm{cm}^{-2}$. The total molecular gas content under the beam $\left(M_{\text {mol }}\right)$ is derived by multiplying $M\left(\mathrm{H}_{2}\right)$ by 1.36 in order to include the Helium mass fraction. Although a variation of the $\chi$ conversion factor cannot be excluded among the observed galaxies, we will take the above derived values as a good estimate of the molecular gas masses.

HI masses $\left(M_{\mathrm{HI}}\right)$ have been taken from various sources or calculated from the $m_{21}$ parameter of LEDA (Paturel et al. 1997), assuming:

$M_{\mathrm{HI}}=2.35 \times 10^{5} \times 10^{-0.4\left(m_{21 \mathrm{c}}-17.4\right)} d^{2}$

with $d$ being the distance in Mpc. Hereafter, $M_{\text {gas }}$ is used for the total mass of cold gas, i.e., $M_{\mathrm{gas}}=M_{\mathrm{mol}}+M_{\mathrm{HI}}$. 
The mass values of X-ray emitting gas $\left(M_{\mathrm{X}}\right)$ have been derived from ROSAT data (Beuing et al. 1999) and from EINSTEIN data (Fabbiano et al. 1992; Burstein et al. 1997), assuming:

$M_{\mathrm{X}}=10^{-24} L_{x}^{0.5} L_{B}^{1.2}$.

Note that this formula (Roberts et al. 1991) is generally valid for early-type galaxies.

Finally, warm dust masses ( $\left.M_{\text {dust }}\right)$ have been calculated from IR fluxes $\left(S_{60}\right.$ and $\left.S_{100}\right)$ published by Knapp et al. (1989) and from LEDA raw data, kindly furnished by G. Paturel, assuming:

$M_{\mathrm{d}}=4.78 \times 10^{-3} S_{100} d^{2}\left(\exp \left(144.06 / T_{\mathrm{d}}\right)-1\right)$

where dust temperature $T_{\mathrm{d}}=49 \times\left(S_{60} / S_{100}\right)^{0.4}$ and $d$ is the distance in Mpc. A more accurate calculation giving the total dust mass, including the coldest component not detected by IRAS, is beyond the scope of this work.

All distance-dependent values available from the literature have been re-scaled to the adopted distances from LEDA, explicitly listed in Table 1.

\section{Observations}

Emission in the $J=1-0$ and $J=2-1$ transitions of ${ }^{12} \mathrm{CO}$ among the sample of 11 galaxies was searched for using the Swedish-ESO Submillimeter Telescope (SEST) at La Silla, equipped with the dual channel IRAM $115 / 230 \mathrm{GHz}$ receivers which allow for simultaneous observations. There were three different observation runs: November 6 th-11th 1998, May 28th-31st 1999 and May 7th-11th 2000. Beam sizes were $45^{\prime \prime}$ and $23^{\prime \prime}$ at $115 \mathrm{GHz}$ and $230 \mathrm{GHz}$, respectively. Unless explicitly stated, the temperature scale used throughout the paper is antenna temperature, corrected from atmospheric losses and rear spillover $\left(T_{\mathrm{a}}^{*}\right)$. When deriving line ratios, it is assumed that main beam efficiencies $\eta_{\text {beam }}(115 \mathrm{GHz})=0.70$ and $\eta_{\text {beam }}(230 \mathrm{GHz})=0.50$, in order to refer temperatures to the main beam brightness scale. Spectrometers cover bandwidths of $995 \mathrm{MHz}$ $\left(1290 \mathrm{~km} \mathrm{~s}^{-1}\right)$ and $543 \mathrm{MHz}\left(1410 \mathrm{~km} \mathrm{~s}^{-1}\right)$ for the $J=$ $2-1$ and $J=1-0$ lines respectively. Dual-beam switching was used, with a beam throw of $12^{\prime}$ to produce a flat baseline. Typical system temperatures ranged from 190$430 \mathrm{~K}$. Pointing and focus were checked every $2-3$ hours, using several $\mathrm{SiO}$ maser sources located near the target galaxy. The rms accuracy of the pointing model was typically $\leq 2^{\prime \prime}$, assuring an absolute positional accuracy better than $5^{\prime \prime}$.

Except for one case (NGC 3497), we made only single point maps centered on the nuclei of the galaxies. Individual scans at each position were coadded to get total integration times ranging from $1 \mathrm{~h}$ to $7 \mathrm{~h}$. Spectra were Hanning-smoothed to a velocity resolution of $30 \mathrm{~km} \mathrm{~s}^{-1}$ (for both lines) with the exception of narrow lines for which a higher resolution was kept (see below). Linear baselines were fitted and subtracted from the smoothed spectra using the GILDAS software package.

\section{Results for the SEST sample}

This section presents the main results of the CO observations for the 11 galaxies of the SEST sample, preceded by a short description of the systems.

\subsection{ESO $263-48$}

This galaxy, also denoted Anon 1029-45, has been included in a list of dust-lane ellipticals by Hawarden et al. (1981). It has a prominent and strongly warped dust lane going across the major axis up to $r=30^{\prime \prime}(5.2 \mathrm{kpc})$. Due to its prominent dust lane which gives the system the appearance of an edge-on disk, it has often been classified as $\mathrm{S} 0$, although it has all the properties (photometric profile, luminosity and size) of giant ellipticals. Furthermore, long exposure plates of the galaxy show no signature of a stellar disk.

The stellar kinematics have been studied by Bertola et al. (1988a), who derived a maximum rotational velocity of $210 \mathrm{~km} \mathrm{~s}^{-1}$ reached at $r=20^{\prime \prime}(3.4 \mathrm{kpc})$ and a velocity dispersion of $260 \mathrm{~km} \mathrm{~s}^{-1}$. Ionized gas in counterrotation, with spin velocities of $\sim 250 \mathrm{~km} \mathrm{~s}^{-1}$ at $r=$ $7^{\prime \prime}(1.2 \mathrm{kpc})$, has been detected along the major axis (Bertola et al. 1988b). There are no HI observations available for ESO263-48; the galaxy also remains undetected in the ROSAT survey. However, this elliptical is particularly rich in dust; from the IRAS fluxes we estimate $M_{\text {dust }} \sim 3.8 \times 10^{5} M_{\odot}$. A continuum radio emission has been detected at 1.4 and $4.9 \mathrm{GHz}$, which extends perpendicularly to the dust lane (Bertola et al. 1988a).

The $J=2-1$ and $J=1-0 \mathrm{CO}$ spectra of Fig. 1 show $\sim 800 \mathrm{~km} \mathrm{~s}^{-1}$-wide emission profiles centered at $v=$ $2810 \mathrm{~km} \mathrm{~s}^{-1}$ (hereafter taken as the CO-based systemic velocity; $\left.v_{\mathrm{sys}}\right)$. The $J=1-0$ spectrum is asymmetrical with respect to $v_{\text {sys }}$ as it can be fitted by three Gaussianlike components. Emission of the two extreme velocity components at $v=2517 \mathrm{~km} \mathrm{~s}^{-1}$ and $v=3170 \mathrm{~km} \mathrm{~s}^{-1}$ can be explained by the presence of an unresolved $\mathrm{H}_{2}$ disk with a rotation speed of $v_{\text {rot }}=325 \mathrm{~km} \mathrm{~s}^{-1}$, reached within $r=2 \mathrm{kpc}$ (the upper limit on $r$ is set by the $J=2-1$ beam). The value of $v_{\text {rot }}$ derived from $\mathrm{CO}$ is significantly larger than that which is inferred for the ionized gas at the same radius $\left(\sim 250 \mathrm{~km} \mathrm{~s}^{-1}\right)$. This discrepancy may indicate that the $\mathrm{H}_{2}$ disk seen in projection extends farther out. The asymmetry in the ${ }^{12} \mathrm{CO}(J=1-0)$ spectrum is caused by the existence of a strong component at $V=2980 \mathrm{~km} \mathrm{~s}^{-1}$, redshifted by $170 \mathrm{~km} \mathrm{~s}^{-1}$ with respect to $v_{\mathrm{sys}}$, and having no blue-shifted counterpart. The latter can come from an asymmetrical distribution in the $\mathrm{H}_{2}$ disk or, alternatively, be the signature of a warp in the molecular gas disk (as suggested by the distorted dust lane).

The molecular gas content under the $J=1-0$ beam can be derived within a radius $r=22^{\prime \prime}(4 \mathrm{kpc})$ (close to the maximum extent of the dust lane feature). It was calculated to be $M_{\mathrm{mol}}=8 \times 10^{8} M_{\odot}$, a high value for an elliptical galaxy. 

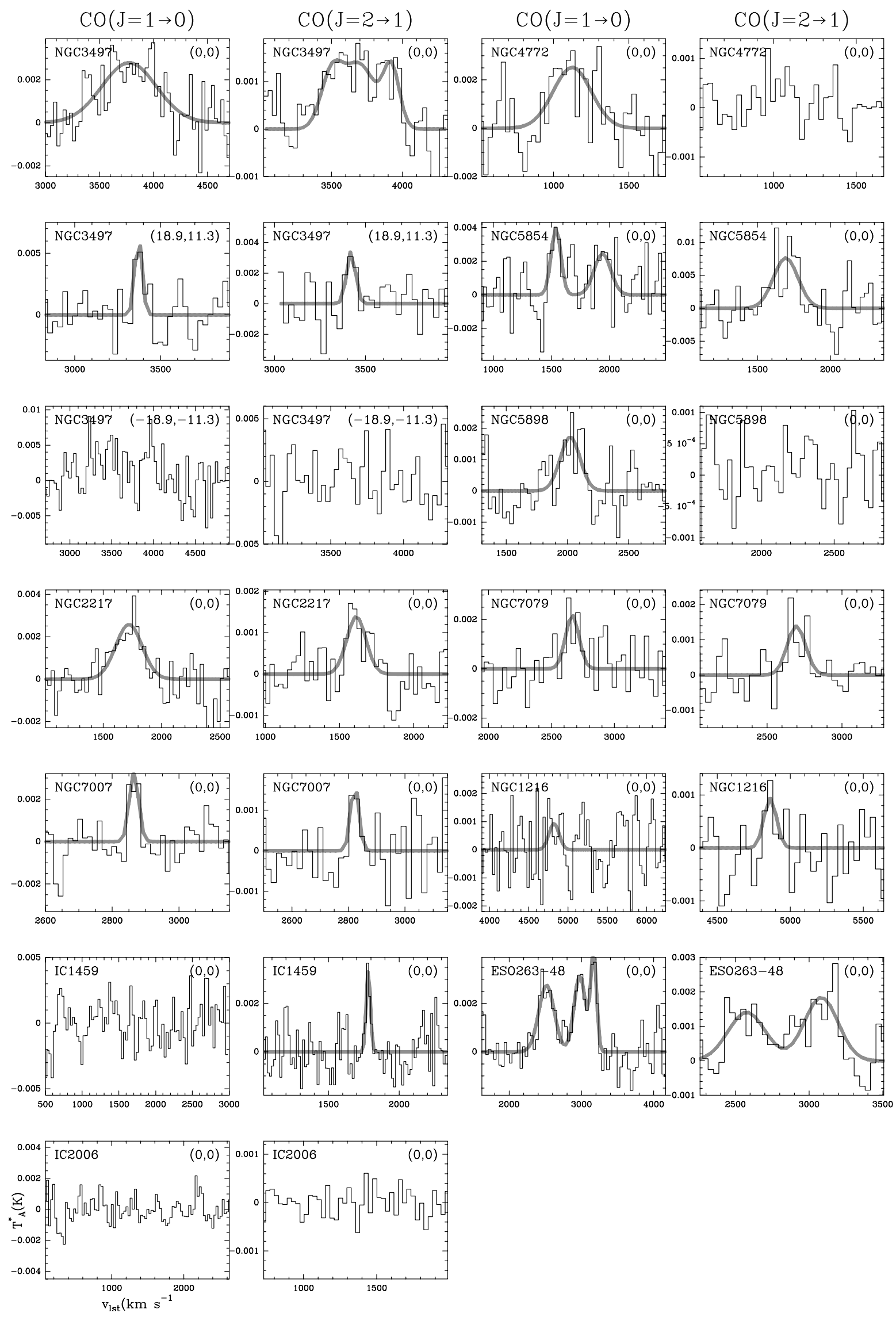

Fig. 1. The $J=1-0$ and $J=2-1$ CO spectra for the galaxies observed by the SEST telescope. Gaussian fits on the lines are shown when available. 


\subsection{IC 1459}

The absolute magnitude and the intrinsic diameter of this galaxy (see Table 1) are both characteristic of a giant elliptical. IC 1459 has a massive counterrotating stellar core ( $M \sim 10^{10} M_{\odot}$, according to Franx \& Illingworth 1988) and its outer stellar isophotes are twisted, an indication that the stellar body is triaxial. This galaxy shows dust absorption in the central 10" (Sparks et al. 1985) and faint pseudo-arms in the outer part $\left(r \sim 3.5^{\prime}\right.$ Malin 1985). The counterrotating core, which hosts a compact radio source (Franx \& Illingworth 1988), has a radius of $\sim 2^{\prime \prime}$ $(200 \mathrm{pc})$ and a projected spin velocity of $170 \pm 20 \mathrm{~km} \mathrm{~s}^{-1}$. On dynamical grounds, the core can be described as a disk, rather than as an ellipsoid. In contrast, the outer directly-rotating stellar body has a slower rotation figure; it reaches $\sim 45 \pm 8 \mathrm{~km} \mathrm{~s}^{-1}$ at $r=40^{\prime \prime}(4 \mathrm{kpc})$. The galaxy is crossed by a disk of ionized gas, whose emission is evenly detected up to $r=35^{\prime \prime}(3.5 \mathrm{kpc})$; the ionized gas rotates in the same direction than the outer stellar body, but at a higher speed (350 $\mathrm{km} \mathrm{s}^{-1}$ ) (Franx \& Illingworth 1988). Therefore counterrotation in this galaxy seems confined to the inner core and affects only the stars. X-ray emission peaks in the galaxy nucleus (Roberts et al. 1991), an indication of central activity.

Walsh et al. (1990) report a negative detection of HI emission, the upper limit on $M_{\mathrm{HI}}$ being $\sim 10^{7} M_{\odot}$. Our ${ }^{12} \mathrm{CO}(J=1-0)$ spectrum also shows a negative detection for molecular gas emission. In contrast, the integrated ${ }^{12} \mathrm{CO}(J=2-1)$ spectrum shows clearly a narrow emission line of $F W H M \sim 35 \mathrm{~km} \mathrm{~s}^{-1}$, centered at $1782 \mathrm{~km} \mathrm{~s}^{-1}$ (see Fig. 1). The velocity centroid of CO is redshifted by $\sim 100 \mathrm{~km} \mathrm{~s}^{-1}$ with respect to the galaxy systemic velocity $\left(v_{\mathrm{sys}}=1691 \mathrm{~km} \mathrm{~s}^{-1}\right)$. This discrepancy of velocity centroids and the narrowness of the ${ }^{12} \mathrm{CO}(J=2-1)$ line both indicate that emission cannot come from a rotating molecular gas disk in equilibrium that could be associated with the ionized disk or, alternatively, with the counterrotating core. In the discarded scenario of a molecular gas disk, the CO line should be $3-5$ times wider than is actually observed, considering the size of our beam. Instead, the CO profile may come from a Giant Molecular Association (GMA). Furthermore, the derived upper limit on the mass of molecular gas, $M_{\mathrm{mol}}<2 \times 10^{7} M_{\odot}$, is noticeably low. Additional support for the interpretation outlined above comes from the high resolution deconvolved $V$-band images of the dust distribution, obtained with the HST. The morphology of the dust lane source in the inner $4^{\prime \prime}$ of the galaxy is very irregular and indicates non equilibrium motions (Forbes et al. 1994). Finally, the estimated $3 \sigma$ upper limit on the $(J=2-1) /(J=1-0)$ ratio $(>1.3)$ suggests that the $\mathrm{CO}$ emission might be partly optically thin.

Similar molecular gas components have been found in other early-type galaxies, such as NGC 404 (Wiklind \& Henkel 1990), classified as a gas accreting elliptical with a minor axis dust lane (Bertola \& Galletta 1978). This GMA may be a residual of one of the galaxies involved in the passed merger that is supposed to be at the origin of IC 1459 (Franx \& Illingworth 1988).

\subsection{IC 2006}

The size and the luminosity of IC 2006 put this galaxy among the dwarf ellipticals. Counterrotation is present in an outer ring of atomic gas, which is aligned with the apparent major axis of the galaxy. At the radius of the HI ring, the galaxy luminosity decreases to $B \sim$ $27 \mathrm{mag} \operatorname{arcsec}^{-2}$ (Schweizer et al. 1989). Schweizer and collaborators describe the HI distribution as a $2 \mathrm{kpc}$ wide circular ring of radius $\sim 11 \mathrm{kpc}$, inclined at $37^{\circ}$. With the adopted parameters, the rotation speed of the HI gas would be $200 \mathrm{~km} \mathrm{~s}^{-1}$ at this distance. HI gas remains undetected inside the ring. In contrast, faint emission from ionized gas is detected within $2.5 \mathrm{kpc}$ of the nucleus, characterized by a velocity gradient which is smaller and also inverted with respect to that of the stars (from $-70 \mathrm{~km} \mathrm{~s}^{-1}(\mathrm{NE})$ to $50 \mathrm{~km} \mathrm{~s}^{-1}$ (SW), relative to $\left.v_{\mathrm{sys}}=1385 \mathrm{~km} \mathrm{~s}^{-1}\right)$. The counterrotating ionized gas disk is highly turbulent, with a measured velocity dispersion of $190 \mathrm{~km} \mathrm{~s}^{-1}$ (Schweizer et al. 1989).

The upper limit set by ROSAT observations (Beuing et al. 1999) indicates that, contrary to IC 1459, IC 2006 has no relevant quantity of hot gas (see Table 1). Based on the optical photometry and the kinematics of the outer HI ring, Schweizer et al. (1989) derive the presence of a dark halo which contains about twice the mass of the luminous stellar body. Our single-point ${ }^{12} \mathrm{CO}(J=1-0)$ map sampled the galaxy nucleus up to $r=3.5 \mathrm{kpc}$. This region includes the ionized gas disk but excludes the outer $\mathrm{HI}$ ring. $\mathrm{CO}$ emission was not detected; the latter implies an upper limit for the central $(r<3.5 \mathrm{kpc})$ molecular gas content of $M_{\text {mol }}<1.4 \times 10^{7} M_{\odot}$.

\subsection{NGC 1216}

This galaxy belongs to the Hickson compact group H 23 . It is an almost edge-on disk galaxy classified as S0-a in the LEDA database. Counterrotation in the ionized gas is detected from optical emission lines (Rubin et al. 1991). The gaseous disk extends up to a radius of $2 \mathrm{kpc}$, with an observed maximum rotational speed of $75 \mathrm{~km} \mathrm{~s}^{-1}$; this velocity is significantly lower than that of the stars at the same radius $\left(\sim 175 \mathrm{~km} \mathrm{~s}^{-1}\right)$. The latter may be an indication that the gaseous disk is either warped or tilted with respect to the galaxy plane. The optical images of this galaxy show no signature of dust absorption. Williams \& van Gorkom (1995) report a negative detection for this galaxy in the $21 \mathrm{~cm}$ line, which gives an upper limit of $M_{\mathrm{HI}}<5 \times 10^{7} M_{\odot}$. IRAS, ROSAT and, finally, our CO observations report negative detections for NGC 1216. The latter give an upper limit of $M_{\text {mol }}<3 \times 10^{8} M_{\odot}$ for the molecular gas content, within a region of radius $r=7 \mathrm{kpc}$. 


\subsection{NGC 2217}

NGC 2217 is a barred spiral (SBa) seen almost face-on. It has an outer stellar+gaseous ring of radius $\sim 8 \mathrm{kpc}$ and an inner oval ring of radius $\sim 4 \mathrm{kpc}$, which encircles the bar.

Bettoni et al. (1990) have studied the distribution and kinematics of the ionized gas, confined to the innermost $20^{\prime \prime}$ ( $\sim 2 \mathrm{kpc}$ ) of the galaxy. The gas distribution suggests the presence of a two-arm spiral structure, whereas the kinematics are characterised by counterrotation with respect to the stars, inside $r \sim 10^{\prime \prime}(\sim 1 \mathrm{kpc})$. However, a detailed analysis of the data by the authors shows that the gas counterrotation is not real, and it may be better accounted for if one assumes the gas to be in a warped disk seen in projection. The ionized gas inside $r=1 \mathrm{kpc}$ would lie in a series of polar rings almost at $90^{\circ}$ with respect to both the bar and the stellar disk. In the outer region $(r=1-2 \mathrm{kpc})$ the plane of the gas rings would have settled towards the disk of the stars, and changed its inclination by nearly 90 degrees. The latter explains why the gas and the stars rotate in the same direction for $r>1 \mathrm{kpc}$. We have classified the system as a polar ring galaxy.

The apparent counterrotation of ionized gas inside $r=1 \mathrm{kpc}$ produces the largest extention of radial velocities along the bar minor axis: from $v=1450 \mathrm{~km} \mathrm{~s}^{-1}$ to $1800 \mathrm{~km} \mathrm{~s}^{-1}$. The $v_{\text {sys }}$ derived from the gas and the star kinematics agree within the margin of errors, being close to $1640 \mathrm{~km} \mathrm{~s}^{-1}$. Bettoni et al. (1990) fit a rotation curve to their data, inferring de-projected rotational velocities of $125 \mathrm{~km} \mathrm{~s}^{-1}$ and $150 \mathrm{~km} \mathrm{~s}^{-1}$ for the stars and the gas, respectively, at a radius $r=1 \mathrm{kpc}$.

HI observations reported by Huchtmeier (1982) show a double-horned emission profile centered at $v_{\text {sys }}=$ $1615 \mathrm{~km} \mathrm{~s}^{-1}$, close to the value found by Bettoni et al. (1990) and with a total width at zero power of $\sim 300 \mathrm{~km} \mathrm{~s}^{-1}$. The re-scaled HI mass is $M_{\mathrm{HI}}=2.7 \times$ $10^{9} M_{\odot}$. As there is no high-resolution HI map of NGC 2217, the location of the HI gas is uncertain.

The spectra in Fig. 1 show the detection of molecular gas emission for the two lines of ${ }^{12} \mathrm{CO}$. The two profiles differ significantly, however. Whereas the $J=1-0$ line is centered at $v=1720 \mathrm{~km} \mathrm{~s}^{-1}$ (i.e., redshifted $100 \mathrm{~km} \mathrm{~s}^{-1}$ with respect to $v_{\text {sys }}$ ), the $J=2-1$ line peaks at $v \sim v_{\text {sys }}$ (as defined above). Although the low spatial resolution of these observations tells us little on the precise location of molecular clouds, the reported asymmetry of the $J=1-0$ profile (which samples the disk up to $r=2 \mathrm{kpc}$ ) suggests that the distribution of $\mathrm{H}_{2}$ gas in NGC 2217 is highly asymmetrical and/or that the kinematics of molecular clouds might depart from circular motions. Most noticeably, the integrated HI profile also shows a pronounced asymmetry. The FWHM of the two CO lines are close to the values found in $\mathrm{HI}$ and in optical emission lines $\left(\sim 250-300 \mathrm{~km} \mathrm{~s}^{-1}\right)$.

We derived a molecular gas content of $M_{\text {mol }} \sim 9 \times$ $10^{7} M_{\odot}$ up to $r=2 \mathrm{kpc}$.

\subsection{NGC 3497}

NGC 3497 is a major-axis dust lane elliptical known by different names (NGC $3525=$ NGC $3528=$ IC 2624). The ringed dusty disk shown in the $B-R$ color maps of Ebneter \& Balick (1985) seems to have the same extent as the stellar disk (diameter $\sim 70^{\prime \prime}$ ). As with all major-axis dustlane ellipticals, e.g. ESO 263-48 in this paper, the dust signature is interpreted to be the result of an accretion episode. The galaxy has a fainter galaxy at $2^{\prime}$ (named NPM1G-19.0362) and a companion with similar redshift at $5^{\prime}(\mathrm{NGC} 3529=\mathrm{IC} 2625)$.

Stellar and gas rotation curves have been derived by Bertola et al. (1988a), who measured a radial velocity difference of $\sim 240 \pm 30 \mathrm{~km} \mathrm{~s}^{-1}$ between the western and eastern sides of the major axis (on the NE side stars are receding). The measured systemic velocity is $v_{\text {sys }}=$ $3672 \pm 25 \mathrm{~km} \mathrm{~s}^{-1}$. No X-ray, IR or HI data are available in the literature.

The emission of both lines of ${ }^{12} \mathrm{CO}$ were observed in three positions located along the major axis of the disk: the $(0,0)$ offset centered on the galaxy nucleus and two off-centered positions at $r= \pm 22^{\prime \prime}$. The $J=2-1$ and $J=$ 1-0 spectra shown in Fig. 1 reveal the presence of molecular gas in the central region (up to $r=5 \mathrm{kpc}$ ) and also the detection of the $J=1-0$ line of ${ }^{12} \mathrm{CO}$ in the NE offset. In the SW position, however, CO emission was not detected. The ${ }^{12} \mathrm{CO}(J=1-0)$ central spectrum is fitted well by a single Gaussian profile, centered at $3774 \mathrm{~km} \mathrm{~s}^{-1}$ and with $F W H M=600 \mathrm{~km} \mathrm{~s}^{-1}\left(\sim 1000 \mathrm{~km} \mathrm{~s}^{-1}\right.$ at zero power $)$; therefore, it is $100 \mathrm{~km} \mathrm{~s}^{-1}$ redshifted with respect to $v_{\text {sys }}$. In contrast, the $J=2-1$ profile shows three velocity components at $v=3497 \mathrm{~km} \mathrm{~s}^{-1}, v=3693 \mathrm{~km} \mathrm{~s}^{-1}$ (close to the optically determined $v_{\mathrm{sys}}$ ) and $v=3497 \mathrm{~km} \mathrm{~s}^{-1}$. The velocity asymmetry in the central $J=1-0$ spectrum may indicate that $\mathrm{H}_{2}$ distribution is slightly asymmetrical in the disk within $r=5 \mathrm{kpc}$.

A comparison between the radial velocity measured on the CO spectrum $\left(\sim 3570 \mathrm{~km} \mathrm{~s}^{-1}\right)$ and the stellar velocities observed in the NE side of the major axis (redshifted with respect to $\left.v_{\text {sys }}\right)$, indicates that molecular gas is counterrotating with respect to the stars.

The molecular gas mass within the central $r=5 \mathrm{kpc}$, derived from the ${ }^{12} \mathrm{CO}(J=1-0)$ integrated intensity, would be $M_{\text {mol }}=1.4 \times 10^{9} M_{\odot}$. The amount of molecular gas detected in the $\mathrm{NE}$ offset is $M_{\mathrm{mol}}=2.7 \times 10^{8} M_{\odot}$.

\subsection{NGC 4772}

Haynes et al. (2000) considers NGC 4772 as a case of apparent counterrotation of the ionized gas versus the stars. The kinematical decoupling of the nuclear ionized gas $\left(r<5^{\prime \prime}=0.3 \mathrm{kpc}\right)$, observed along both the minor and the major axes, has been interpreted as the signature of a misaligned embedded gas bar, rather than as evidence of counterrotation. However, this Sa galaxy shares many features with other prototypical counterrotators. Mimicking NGC 3626, the HI content of NGC 4772 is distributed in 
two separate rings, probably non coplanar. As is the case for NGC 3626, the central region of NGC 4772 is HI-poor. Furthermore, the deep optical photometry of the galaxy reveals the presence of a round, low surface brightness disk in the outer part, reminiscent of a similar feature reported by Buta et al. (1995) in the Sab counterrotator NGC 7217.

The $J=1-0$ spectrum of ${ }^{12} \mathrm{CO}$ (Fig. 1) reveals the presence of molecular gas (inside $r=1.5 \mathrm{kpc}$ ). The line profile, centered at $\sim 1120 \mathrm{~km} \mathrm{~s}^{-1}$ and with $F W H M \sim$ $300 \mathrm{~km} \mathrm{~s}^{-1}$, is slightly redshifted with respect to the optically determined $v_{\text {sys }}=1040 \mathrm{~km} \mathrm{~s}^{-1}$ (the same as derived from $\mathrm{HI})$.

Emission in the $J=2-1$ line is undetected, however. The molecular gas mass within the central $r=1.5 \mathrm{kpc}$, derived from the ${ }^{12} \mathrm{CO}(J=1-0)$ integrated intensity, would be $M_{\mathrm{mol}}=5.4 \times 10^{7} M_{\odot}$.

\subsection{NGC 5854}

NGC 5854 is an early spiral (Sa) characterized by a low gas content and the absence of current star formation. Haynes et al. (2000) have studied the stellar kinematics, using $\mathrm{H} \alpha$ and MgIb optical absorption lines, and the kinematics of ionized gas, using the $\mathrm{N}[\mathrm{II}]$ and $\mathrm{O}[\mathrm{III}]$ optical emission lines. These data reveal the existence of a counterrotating gas disk extending up to $r \sim 7^{\prime \prime}(0.8 \mathrm{kpc})$, with a total velocity range of $120 \mathrm{~km} \mathrm{~s}^{-1}$. The stellar velocities measured at $r \sim 40^{\prime \prime}(4.5 \mathrm{kpc})$ reach $\pm 160 \mathrm{~km} \mathrm{~s}^{-1}$. Although $\mathrm{HI}$ content is low, Magri (1994) detected a signal in the nucleus. The HI profile is centered at $166 \mathrm{~km} \mathrm{~s}^{-1}$, close to the optically determined value for $v_{\text {sys }}=1669 \pm 30 \mathrm{~km} \mathrm{~s}^{-1}$ (Fouque et al. 1992). The narrowness of the HI spectrum ( FWHM $\sim 100 \mathrm{~km} \mathrm{~s}^{-1}$, namely, less than the measured stellar velocity spread) suggests a close association of the HI component with the counterrotating ionized gas (see discussion in Haynes et al. 2000).

Although weak, the ${ }^{12} \mathrm{CO}(J=1-0)$ spectrum shows the existence of $\mathrm{H}_{2}$ gas within $r=2.3 \mathrm{kpc}$. There is a hint of a double-horned profile, with two velocity components at $v=1539 \mathrm{~km} \mathrm{~s}^{-1}$ (with $F W H M \sim 100 \mathrm{~km} \mathrm{~s}^{-1}$ ) and $v=1930 \mathrm{~km} \mathrm{~s}^{-1}$ (with $F W H M \sim 170 \mathrm{~km} \mathrm{~s}^{-1}$ ), equidistant from $v=1735 \mathrm{~km} \mathrm{~s}^{-1}$. The $\mathrm{CO}$ spectrum in the $J=2-1$ line confirms the detection of molecular gas. Not surprisingly, the $J=2-1$ and $J=1-0$ profiles differ. The $J=2-1$ line shows hints of two velocity components, although with a smaller velocity spread $\left(v=1630 \mathrm{~km} \mathrm{~s}^{-1}\right.$ and $\left.1740 \mathrm{~km} \mathrm{~s}^{-1}\right)$ and is centered at $v \sim 1690 \mathrm{~km} \mathrm{~s}^{-1}$, in reasonable agreement with HI. However the larger velocity spread of the $J=1-0$ spectrum would suggest that, compared to the counterrotating ionized gas core, the $\mathrm{H}_{2}$ disk may extend farther out. The molecular gas mass within the central $r=2.3 \mathrm{kpc}$ was derived from the ${ }^{12} \mathrm{CO}(J=1-0)$ integrated intensity giving; $M_{\mathrm{mol}}=1.6 \times 10^{8} M_{\odot}$.

\subsection{NGC 5898}

NGC 5898 was studied by Bettoni (1984) and Bertola \& Bettoni (1988), who discovered the first case of ionized gas counterrotation in a dust lane elliptical in this galaxy. Their data, which extended out to $\sim 10^{\prime \prime}(1.4 \mathrm{kpc})$, have recently been completed by Caon et al. (2000) who analysed the stellar and the gas kinematics farther out (up to $\left.r \sim 35^{\prime \prime}(4.8 \mathrm{kpc})\right)$. The new data along the major axis show the existence of a stellar core of radius $r \sim 5^{\prime \prime}$ $(0.7 \mathrm{kpc})$ which counterrotates with respect to the outer stellar body. The ionized gas counterrotates with respect to the inner stellar core, and it therefore corotates with the outer stars. In contrast, gas is seen to counterrotate at all radii along the minor axis. This might indicate that angular momentum vectors of the ionized gas and the stars are certainly misaligned, but not antiparallel. The observations of this galaxy at X-ray and IR wavelengths show an upper limit for hot gas of $\sim 3 \times 10^{8} M_{\odot}$ and a moderate quantity of dust, of a few $10^{4} M_{\odot}$.

The $J=1-0$ spectrum of ${ }^{12} \mathrm{CO}$ (Fig. 1 ) shows a tentative detection of molecular gas inside $r=3.1 \mathrm{kpc}$. The line profile, centered at $\sim 2020 \mathrm{~km} \mathrm{~s}^{-1}$ and with $F W H M \sim$ $190 \mathrm{~km} \mathrm{~s}^{-1}$, is slightly blueshifted with respect to the optically determined value of $v_{\text {sys }}\left(\sim 2100 \mathrm{~km} \mathrm{~s}^{-1}\right.$, derived from Bertola \& Bettoni 1988). The observed asymmetry might arise if the $\mathrm{H}_{2}$ gas was associated with the ionized disk, which shows a marked extension towards the SW (where ionized gas radial velocities are blueshifted). In this scenario molecular gas would also be counterrotating.

The ${ }^{12} \mathrm{CO}$ emission is undetected in the $J=2-1$ line, however. We have calculated the molecular gas mass within the central $r=3.1 \mathrm{kpc}$, using the ${ }^{12} \mathrm{CO}(J=1-0)$ integrated intensity, giving $M_{\mathrm{mol}}=10^{8} M_{\odot}$.

\subsection{NGC 7007}

The optical images of NGC 7007 show an elliptical-like body encircled by an off-centered bow-shaped dust lane on the eastern side. Dettmar et al. (1990) discovered, in this galaxy, the signature of a counterrotating ionized gas disk by comparing the spectrograms of gas emission (NII $\lambda 6853)$ and stellar absorption lines $\left(\mathrm{H}_{\alpha}\right)$. Spectra taken later (Bettoni et al. 2001a) allowed a detailed analysis of the stellar and the gas kinematics, characterised by maximum rotational velocities of $\pm 150 \mathrm{~km} \mathrm{~s}^{-1}$ and $\pm 175 \mathrm{~km} \mathrm{~s}^{-1}$ respectively, reached at $r=10^{\prime \prime}$. The central velocity dispersion for stars is $150 \mathrm{~km} \mathrm{~s}^{-1}$, whereas gas lines have instrumental width $\left(<100 \mathrm{~km} \mathrm{~s}^{-1}\right)$. The galaxy contains a source of infrared emission detected by IRAS, and at X-ray wavelengths the published work reports an upper limit (see Table 1).

Our $J=2-1$ and $J=1-0$ spectra both show a weak narrow line of $\sim 30 \mathrm{~km} \mathrm{~s}^{-1} F W H M$, centered at $\sim 2850 \mathrm{~km} \mathrm{~s}^{-1}$, close to the optical redshift of $2924 \pm$ $66 \mathrm{~km} \mathrm{~s}^{-1}$ reported in RC3 (de Vaucouleurs et al. 1991). These results are, however, at odds with that quoted by Da Costa et al. (1991) (3053 $\left.\pm 20 \mathrm{~km} \mathrm{~s}^{-1}\right)$, who estimated 
$v_{\text {sys }}$ as a weighted average between gas emission and stellar absorption data. If the Da Costa et al. (1991) value is more accurate, as indicated by the additional spectra of Bettoni et al. (2001a), the reported difference between the CO peak and the optical systemic velocity could be explained with an asymmetry in the distribution of cold gas. The derived molecular gas mass, $\sim 6 \times 10^{7} M_{\odot}$, could be well accounted for if the emission observed came from a few Giant Molecular Associations (GMAs) in the center of the galaxy, as observed in IC 1459. The narrowness of lines in both transitions supports this scenario.

\subsection{NGC 7079}

NGC 7079 is a weakly barred SB0 galaxy, a member of an interacting pair. Bettoni \& Galletta (1997) detected a counterrotating disk-like structure of ionized gas which extends up to a radius of $r=2 \mathrm{kpc}$. The radial velocities for the gas span from $2600 \mathrm{~km} \mathrm{~s}^{-1}$ to $2800 \mathrm{~km} \mathrm{~s}^{-1}$. The stellar kinematics is typical of an undisturbed disk. The measured radial velocities (up to $r=4.5 \mathrm{kpc}$ ) range from $v \sim 2500 \mathrm{~km} \mathrm{~s}^{-1}$ to $v \sim 2900 \mathrm{~km} \mathrm{~s}^{-1}$ and give a $v_{\text {sys }}=2680 \mathrm{~km} \mathrm{~s}^{-1}$ and a central velocity dispersion of $150 \mathrm{~km} \mathrm{~s}^{-1}$. No X-ray emission has been detected from this galaxy; the IRAS satellite detected infrared emission.

The $J=2-1$ and $J=1-0$ lines of CO are detected in this galaxy, showing the presence of molecular gas (see Fig. 1). The line profiles, $\sim 170 \mathrm{~km} \mathrm{~s}^{-1}$ wide at zero power, are centered on the galaxy systemic velocity, derived from optical data. The linewidths of both CO lines agree satisfactorily with the velocity range measured for the counterrotating ionized gas. In contrast, the $\mathrm{CO}$ widths are much smaller than the velocity interval measured in the stellar lines. This may indicate that $\mathrm{H}_{2}$ gas is confined to the inner portion of the galaxy and that it shares the same kinematics as the counterrotating ionized gas. The inferred molecular gas content is $M_{\text {mol }} \sim 1.2 \times 10^{8} M_{\odot}$ up to $r=2 \mathrm{kpc}$.

\section{The enlarged sample of accreting galaxies}

The newly acquired data described above would nevertheless be insufficent to extrapolate estimates on the global gas content to all counterrotators. In order to improve the SEST sample on statistical grounds we added the published data from those counterrotators (Galletta 1996; Kannappan \& Fabricant 2001) with an estimate from any of the different gas tracers: $M_{\mathrm{mol}}, M_{\mathrm{HI}}, M_{\mathrm{dust}}$ or $\mathrm{M}_{\mathrm{X}}$. Masses are derived with the same assumptions used for the SEST sample. The properties of the enlarged sample are in first part of Table 1, together with the list of references relevant for this compilation. This new sample allows a complete study of the global gas content of counterrotators, using different gas tracers in a statistically significant sample of 58 objects.

In order to compare counterrotators and polar rings, the available data on a sample of 46 polar ring galaxies have also been compiled (see Ref. in Table 1). Data include
36 polar ring lenticulars and spirals (Schweizer et al. 1983; Whitmore et al. 1990 and this work), and 10 polar ring ellipticals, known in the literature as ellipticals with minoraxis dust-lanes, such as NGC 5128 (Bertola \& Galletta 1978). Polar ring ellipticals have traditionally been classified as S0s due to the presence of a dark ring or disk in optical pictures. However, the luminosity profiles of these galaxies do not follow an exponential law, typical of stellar disks, but rather a $r^{1 / 4}$ law, characteristic of spheroidal systems. We have therefore re-classified these galaxies as ellipticals, whenever they appear as S0s in catalogs. Not all polar ring lenticulars and spirals present in Whitmore et al. (1990)'s catalogue were finally included in our list. We only selected those systems where the perpendicularity between the ring and the stellar body is clearly visible in the images, discarding all systems appearing doubtful in our inspection of the catalogue. Altogether the sample of accreting systems includes 104 objects.

\section{Building up a comparison sample}

The main aim of this paper is to study the molecular gas content of accreting galaxies (counterrotators and polar rings) and compare it with the average value for normal non-interacting galaxies as a function of the Hubble type. The first non-obvious task is the definition of a comparison sample of normal galaxies. The sample should contain a statistically significant number of objects. This requirement is critical for early-type galaxies, as the majority of accreting systems are of types earlier than Sa (morphological type code $t=1$ ). Moreover, the sample should avoid the inclusion of abnormal objects, suspected to be interacting and/or merging galaxies, e.g those reported in Arp's catalogs.

In the past, two different research groups have built up comparison samples in order to study the variation of the gas content of galaxies along the Hubble sequence: Bregman et al. (1992) and Casoli et al. (1998). Bregman et al. (1992) derived the content of molecular gas, HI, X-ray emitting gas and dust, working on a sample of 467 early-type galaxies, ranging from pure ellipticals ( $\mathrm{E}$, $t=-5$ ) to early spirals ( $\mathrm{Sa}, t=1)$. In their analysis they favoured the use of the total blue luminosity $\left(L_{B}\right)$ of galaxies as the necessary normalisation factor, i.e., the inferred numbers being $M_{\mathrm{mol}} / L_{\mathrm{B}}, M_{\mathrm{HI}} / L_{\mathrm{B}}, M_{\mathrm{dust}} / L_{\mathrm{B}}$ and $M_{\mathrm{X}} / L_{\mathrm{B}}$. They concluded, first, that the gas content of elliptical galaxies is dominated by the hot $\operatorname{phase}\left(M_{\mathrm{X}}>\right.$ $\left.M_{\mathrm{mol}}+M_{\mathrm{HI}}\right)$ and secondly, that $M_{\mathrm{mol}} / L_{\mathrm{B}}$ and $M_{\mathrm{HI}} / L_{\mathrm{B}}$ both show a strong positive gradient from $\mathrm{E}$ to Sa-type systems. However, the number of early type galaxies detected either in $\mathrm{H}_{2}$ or in $\mathrm{HI}$ gas is scarce: for $\mathrm{H}_{2}, 1$ E-type detected with 11 upper limits (hereafter UL) and 6 SOs detected with 18 UL. Poor statistics cast some doubt on their conclusions.

Casoli et al. (1998) discussed the molecular and atomic gas content for a set of 582 objects, mainly disk galaxies, normalizing the gas masses by $D_{25}^{2}$. For comparison, numbers for $\mathrm{H}_{2}$ are: $3 \mathrm{E}$ detected and 7 SOs detected (with 

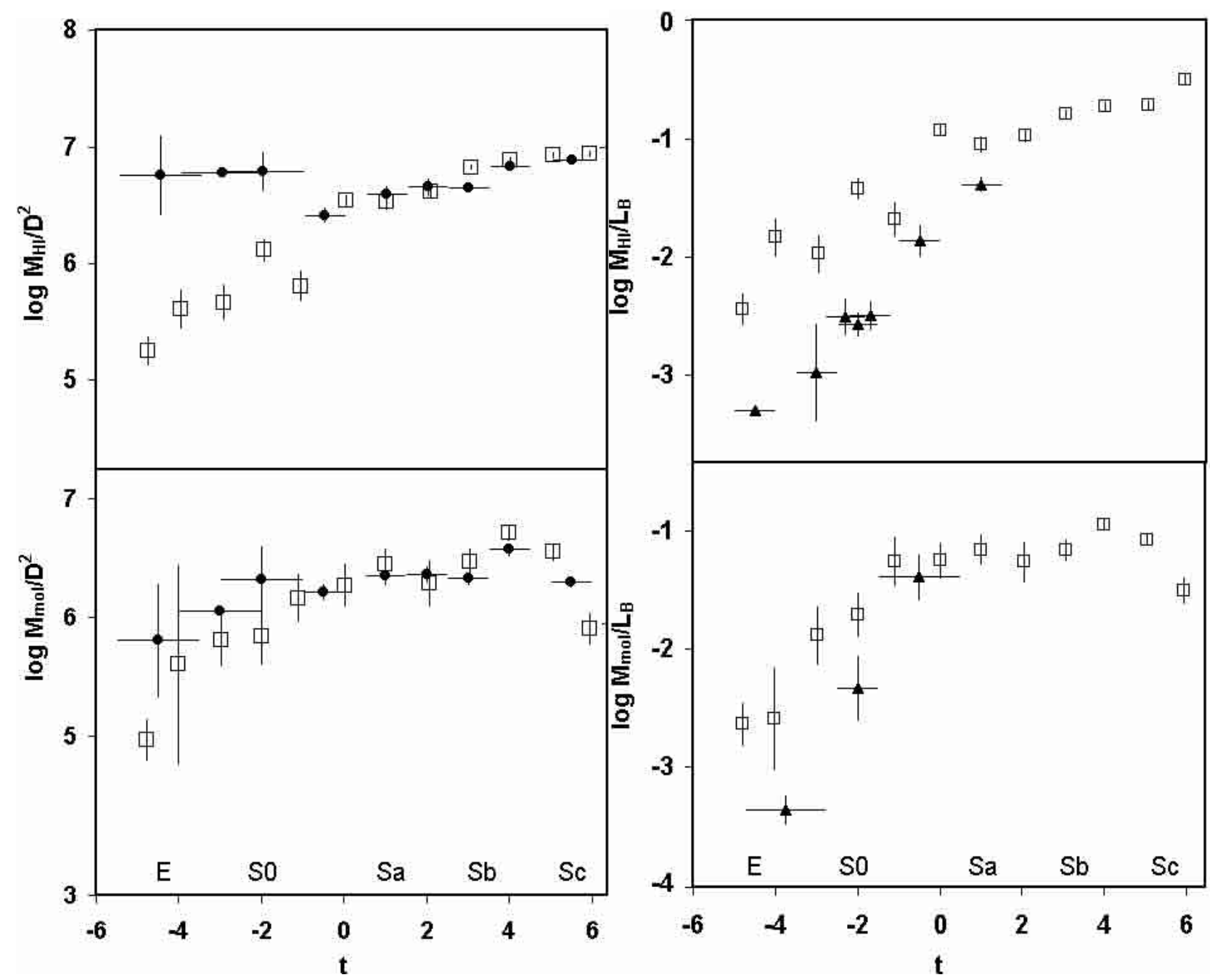

Fig. 2. (Left): $\log M / D^{2}$ ratios in normal galaxies derived for the atomic and molecular gas, from the samples of Casoli et al. (1998) (filled circles) and Bettoni et al. (2001b) (open symbols). (Right): Log $M / L$ ratios in normal galaxies for the atomic and molecular gas, from Bregman et al. (1992) (filled triangles) and Bettoni et al. (2001b) (open symbols).

2 UL). Results from Casoli et al. (1998) are noticeably at odds with that of Bregman et al. (1992): the sharp increase of gas content from $t=-5$ to $t=1$ reported by Bregman et al. (1992) (1-2 orders of magnitude) is not confirmed using Casoli et al. (1998) data. This discrepancy is illustrated in Fig. 2 where the mean values are represented as a function of $t$ for the two samples. To reconcile these discrepant trends one must assume an unrealistic decrease by 2 orders of magnitude in $L_{\mathrm{B}} / D_{25}^{2}$, going from $\mathrm{E}$ to Sa systems. Results from both samples for types $t<1$ are however dubious, considering the poor statistics in this range. Moreover, the two samples include a non-negligible percentage of interacting galaxies (estimated to be close to $\sim 20 \%$ in both samples). Interacting galaxies should be discarded when putting together a comparison sample of normal non-interacting objects.

These limitations were the motivation to build up a new comparison sample of normal galaxies. Paper II by Bettoni et al. (2001b) will discuss extensively the details of this compilation. The over-all numbers give a grand total of 1773 normal galaxies selected from a processed sample of 3800 objects. Bettoni et al. (2001b) purposedly excluded from the selected sample those galaxies belonging to the interacting or disturbed categories (most of them appearing in Arp 1966; Vorontsov-Velyaminov 1959; Arp \& Madore 1987 catalogues). Galaxies listed in the Veron-Cetty \& Veron (2000) catalogue of AGN systems have also been excluded because in some cases their peculiar activity has been attributed to gas accretion. We have taken from Bettoni et al. (2001b) the normalized values $M / L_{B}$ for the molecular, atomic and X-ray emitting gas, as well as for the warm dust content inferred from IRAS. The global statistics for detections (and UL) are: 247 in $\mathrm{H}_{2}$ (113 UL), 774 in HI (149 UL), 196 in X-rays (661 UL) and 861 in IR (555 UL). This sample improves the statistics for early type galaxies compared to previous works, the numbers for $\mathrm{H}_{2}$ being: 10 E-type detected (plus $18 \mathrm{UL}$ ) and 10 lenticulars detected (plus $17 \mathrm{UL}$ ). Note that the galaxies used to build the mean values for the different ISM tracers are not always the same; however, the majority of galaxies in our sample (1135) have detections or upper limits in at least two wavebands. 
We have applied a survival analysis method to the different ensembles of $M / L_{B}$ data. This analysis tool takes properly into account both detections and UL in order to derive representative averages. The mean values are derived and plotted under the label "normal galaxies", and are binned according to the morphological type code (with $\Delta t=1)$. Most noticeably, UL lower significantly the estimated mean $M / L_{B}$ values for normal galaxies of early types (see Bettoni et al. 2001b). The derivation of mean values of molecular gas content from Casoli et al. (1998) used survival analysis also. For HI data, all the galaxies of their sample were detected and so no survival analysis needs to be applied. Also, the analysis of Bregman et al. (1992) takes into account the different detection rates of the various morphological types, but using different nonparametric tests, based on rank.

At first sight, the comparison between the mean values derived from these three different studies (Bregman et al. 1992; Casoli et al. 1998; Bettoni et al. 2001b) shows that $\log \left(M_{\mathrm{HI}} / D_{25}^{2}\right)$ and $\log \left(M_{\mathrm{mol}} / D_{25}^{2}\right)$ of Bettoni et al. (2001b) are intermediate between Casoli et al. (1998) and Bregman et al. (1992) values. Although the cold gas content increases by a factor $\sim 10$ from $\mathrm{E}$ to Sa-types, this gradient is less steep than that reported by Bregman et al. (1992) (see Fig. 2).

To identify any potential bias in the galaxy samples compared in this work (normal galaxies, counterrotators and polar rings), we have analysed the statistical distribution of the following intrinsic properties: $M_{B}, D_{25}$ and FIR flux (given by $m_{\text {FIR }}$ ). Kolgomorov-Smirnov tests applied to these quantities indicate that the distributions of $M_{B}, D_{25}$ and $m_{\mathrm{FIR}}$ are not significantly different for the 3 samples, at a confidence level better than $95 \%$.

\section{The ISM of gas accretors}

We discuss in this section the results obtained from the comparison of the gas/dust content of gas-accreting and normal galaxies. Mean values of $\log M / L$ were obtained for each morphological type, using a $\Delta t=1$ code binning. We studied the deviations from the reference values issued from the survival analysis method applied to normal galaxies (see above). The statistical significance of any difference found between the samples was evaluated by a Student t-test applied to the mean of the values of $\log M / L$, binned according to morphological type. Figures 3-8 illustrate this comparison, whose results can be summarised as follows:

- $\log \left(M_{\mathrm{HI}} / L_{\mathrm{B}}\right)$ and $\log \left(M_{\mathrm{mol}} / L_{B}\right)$ show a large dispersion for accretors of types $t<0$ (Figs. 3 and 4 ). This result holds for counterrotators and polar rings. The values of the gas content for normal galaxies are also highly dispersed for $t<0$;

- Polar ring spirals and lenticulars have a HI content $\sim 1$ order of magnitude higher than normal galaxies (Fig. 3). The reported difference between the samples is established with a $99 \%$ statistical significance. In

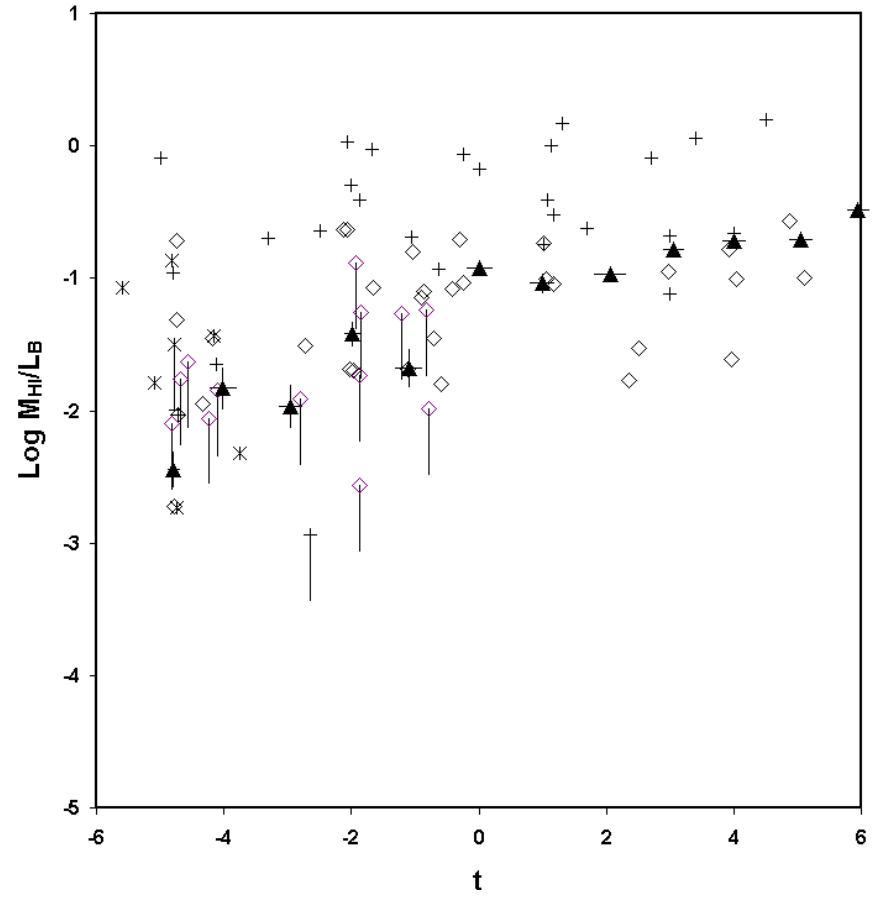

Fig. 3. Plot of Log $M_{\mathrm{HI}} / L_{B}$ versus the morphological type for the samples of counterrotating galaxies (open romboids), polar ring S0s and spirals (crosses) and polar ring ellipticals (asterisks). Filled triangles represent the reference values derived from the comparison sample of Bettoni et al. (2001b).

contrast, there is no significant difference between normal galaxies and polar ring ellipticals regarding the $\mathrm{HI}$ content. This result, previously found and discussed by Richter et al. (1994) and Huchtmeier (1997) is still valid when $\log \left(M_{\mathrm{HI}} / D^{2}\right)$, instead of $\log \left(M_{\mathrm{HI}} / L_{B}\right)$, is used as the gas content estimator. Therefore, this tendency cannot be attributed to any colour bias unexpectedly affecting PRs.

On the other hand, the HI content of counterrotating galaxies shows no significant departure from the expected normal value (Fig. 3);

- The molecular gas content of polar ring galaxies lies above normal values for all galaxy types (Fig. 4). On average, it is calculated that $M_{\mathrm{mol}} / L_{B}$ is $\sim 1$ order of magnitude higher in polar rings; this result, which agrees satisfactorily with the conclusions of Galletta et al. (1997), is established with a $98 \%$ statistical significance. Therefore, the global content of cold gas $\left(M_{\text {gas }}\right)$ in S0/S-polar rings lies $>1$ order of magnitude above standard values with a $99 \%$ certainty (see Fig. 5). On the other hand, the $M(\mathrm{~mol}) / M_{\mathrm{HI}}$ ratio in polar rings stays close to normal values for all types (Fig. 6).

The molecular gas content of counterrotating galaxies is marginally lower than normal for types $t<0$ (Fig. 4). This deficiency is not firmly established, its statistical significance being low $(78 \%)$. In contrast, counterrotating galaxies reach normal $\mathrm{H}_{2}$ masses for $t>0$. Contrary to polar rings, the global content of cold gas in counterrotators, given by $\log \left(M_{\text {gas }} / L_{B}\right)$, 


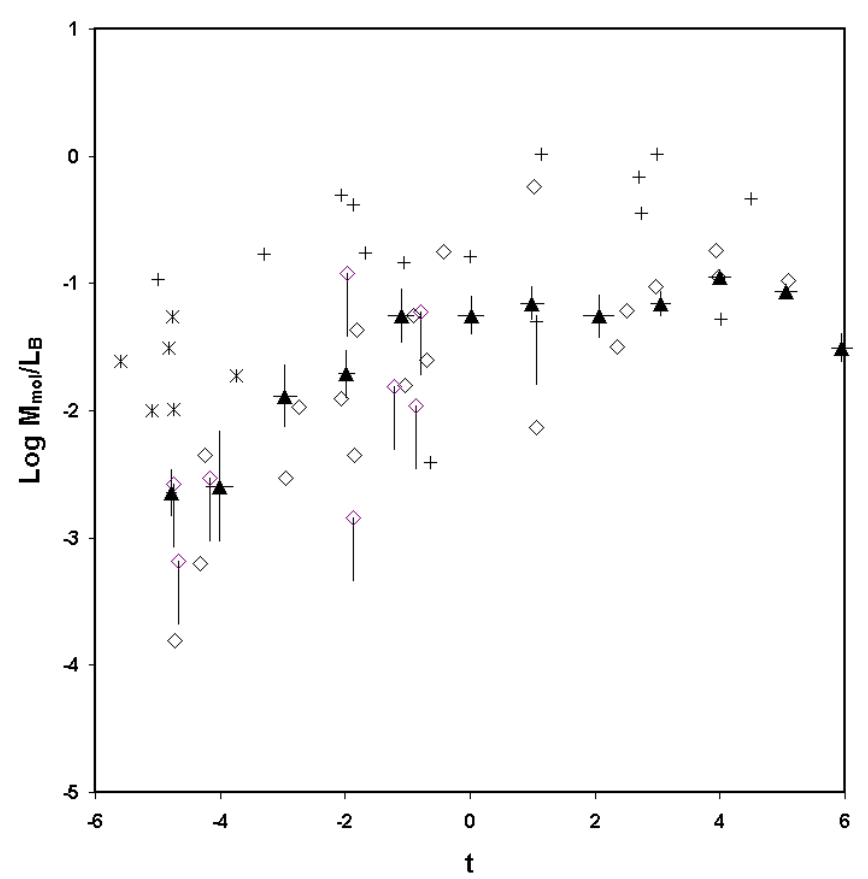

Fig. 4. Variation of the molecular gas masses with the morphological type for the samples of counterrotating galaxies (open romboids), polar ring S0s and spirals (crosses) and polar ring ellipticals (asterisks). Filled triangles represent the reference values derived from the comparison sample of Bettoni et al. (2001b).

shows no relevant departures from normal values for all types (Fig. 5).

However, the HI phase seems to dominate in early type counterrotators $(t<0)$. We tentatively identify an increase by $\sim 1$ order of magnitude in the $M\left(\mathrm{H}_{2}\right) / M_{\mathrm{HI}}$ ratio from $t=-6$ to $t=6$, a factor significantly larger than that observed in normal galaxies (Fig. 6). The latter indicates that some mechanism favouring the transformation of $\mathrm{HI}$ into $\mathrm{H}_{2}$ might be at work for counterrotators on the right-side of the Hubble sequence (see below);

- As derived from $\log \left(M_{\text {dust }}\right) / L_{B}$, polar ring galaxies have a dust content $\sim 0.5-1$ order of magnitude higher than normal. This result is established with a $99 \%$ statistical significance for spirals and lenticulars, whereas it is only at a $90 \%$ certainty level for ellipticals. In contrast, galaxies with counterrotation have a warm dust content not significantly different to normal galaxies for all types (Fig. 7);

- One fifth of counterrotators and nearly half of the polar ring ellipticals have been detected in X-rays. On average, the estimated masses of hot gas (see Table 1) are slightly lower than the ones of normal galaxies. Moreover, the slope fitted to the 17 detections in the $L_{\mathrm{X}}-L_{\mathrm{B}}$ diagram $(\sim 1.7$, Fig. 8$)$ lies between the prototypical value for emission mainly due to hot diffuse gas ( $L_{\text {gas }}$, with slope $\sim 2$ ), and the one for emission being dominated by discrete sources $\left(L_{\text {discr }}\right.$, with $\sim 1$; see Ciotti et al. 1991 for details). Furthermore, $L_{\mathrm{X}}$ values

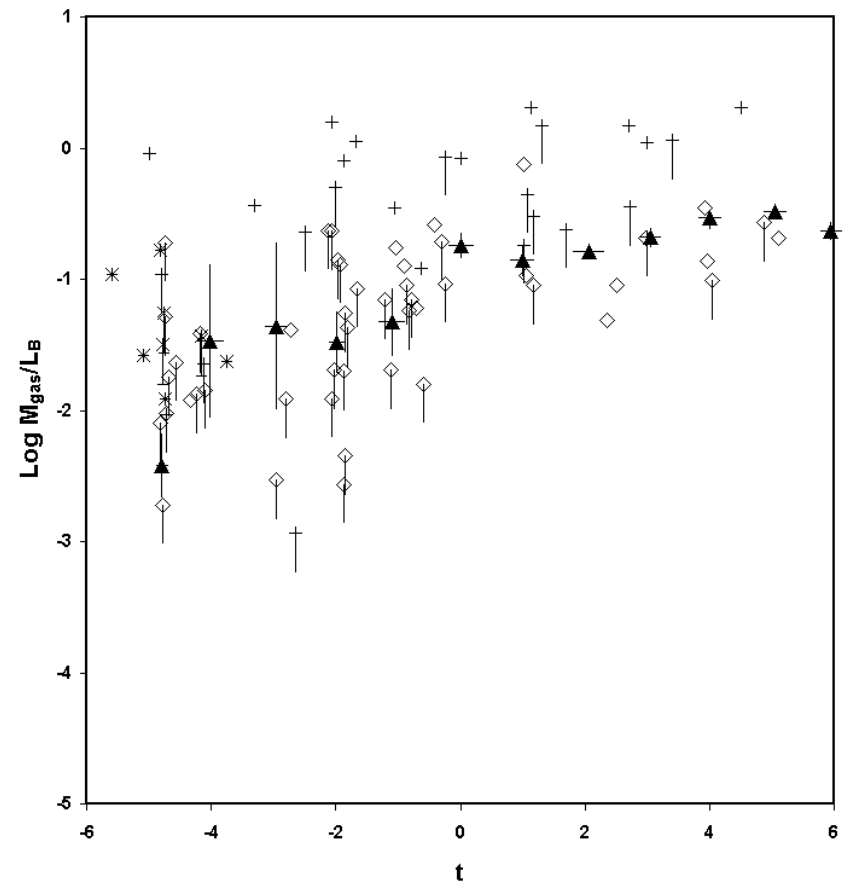

Fig. 5. Change of the cold gas content (molecular and atomic)with respect to morphological type for the samples of counterrotating galaxies (open romboids), polar ring S0s and spirals (crosses) and polar ring ellipticals (asterisks). Filled triangles represent the reference values derived from the comparison sample of Bettoni et al. (2001b).

for accretors are below the emission level predicted by cooling flows models, assuming the recent SN I rate equal to 0.18 (i.e., $1.8 \times 10^{-3} \mathrm{SN}$ I events per year per unit of $\left.10^{10} L_{B} / L_{\odot}\right)$ (Cappellaro et al. 1999). We can conclude that the observed normalised X-ray luminosity of accretors needs no huge starburst event as an explanation;

- A source of uncertainty for $M_{\mathrm{mol}}$ comes from the undersampling of some of the sources. Apart from NGC 3497, only single point maps centered in the galaxy nuclei are available from the SEST observations. Therefore, the derived $M_{\text {mol }}$ should be taken as a lower limit for the SEST sample, as well as for some of the galaxies in the enlarged sample of accretors for which no complete maps are available. Although it is difficult to evaluate accurately the bias introduced on $M_{\text {mol }}$, we are confident to have on average $\sim 70 \%$ of the total molecular gas masses under the SEST beam for spirals. This estimate is based on the comparison between the mean ratios of $D_{\text {beam }} / D_{25}(\sim 0.4$, where $\left.D_{\text {beam }}=22^{\prime \prime}\right)$ for the SEST galaxies and the predicted ratios of $D_{\mathrm{CO}} / D_{25} \sim 0.5$ for typical spiral galaxies. The values of $D_{\mathrm{CO}}$, defined as the diameter of the canonical distribution of $\mathrm{CO}$ in Virgo spirals which contains $70 \%$ of the total CO flux (Kenney \& Young 1988), indicate that molecular gas is highly concentrated in the inner optical disks. Therefore, the reported differences (a factor of $\sim 10$ !) in the molecular gas content between polar rings and counterrotators 


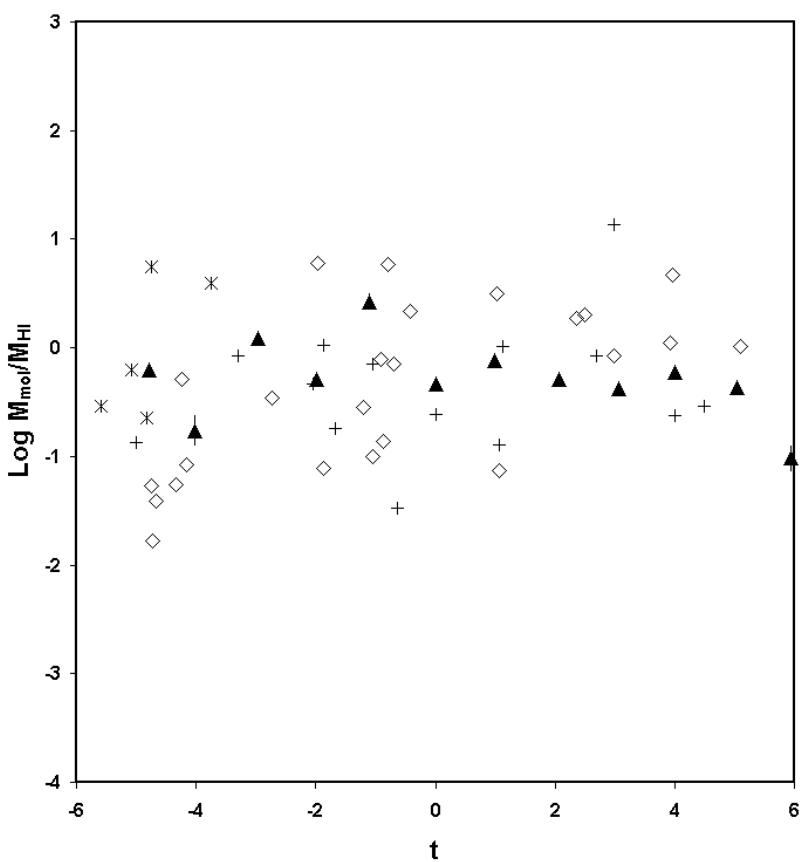

Fig. 6. The ratio of molecular to atomic gas versus the morphological type. Symbols are: counterrotating galaxies (open romboids), polar ring S0s and spirals (crosses), polar ring ellipticals (asterisks) and normal galaxies (filled triangles).

can hardly be attributed to a systematic undersampling of counterrotators, considering also that undersampling in $\mathrm{CO}$ maps affects polar ring galaxies to a comparable extent. Furthermore, results obtained using HI as a tracer of cold gas (much less affected by undersampling), confirm a similar trend as that shown by $\mathrm{CO}$ : polar rings have a gas content significantly higher than counterrotators.

\section{Discussion and conclusions}

Gas and stars along polar orbits can be explained as the result of the acquisition of cold infalling gas by an accreting galaxy. The accreted gas can smear out into a ring after a few orbital periods. The fate of this ring will depend on its orientation, relative to the mass distribution of the accretor, and most importantly, on the mass/selfgravity of the gas. A polar ring may form after a high angle impact with gas which has a spin perpendicular to the equatorial plane of the accretor, remaining in an equilibrium configuration for several Hubble times. In contrast, counterrotating galaxies may form after a low angle impact with a gas disk which has a spin antiparallel to that of the accreting system.

In the most general case, however, the impact angle is intermediate between polar and planar. In this case the orbits of gas clouds will experience differential precession in the non-spherical potential of the galaxy, characterized by a quadrupole component (Steiman-Cameron \& Durisen 1982). The latter applies for disk galaxies (axisymmetric) and ellipticals (triaxials). Sparke (1986) and Arnaboldi \&

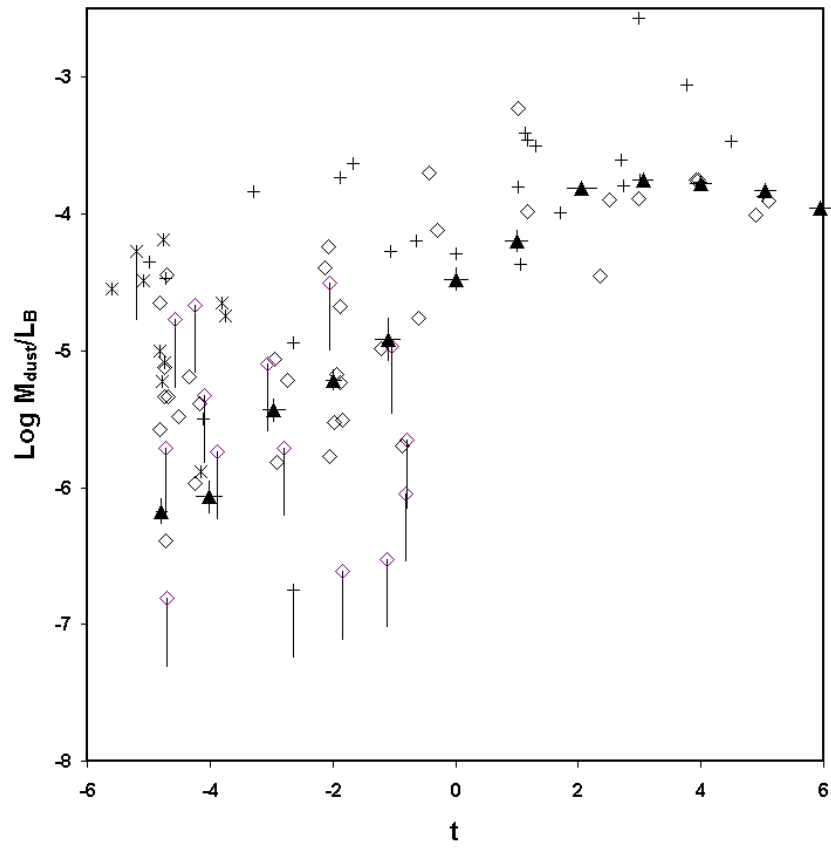

Fig. 7. Variation of the warm dust content with respect to morphological type for the samples of counterrotating galaxies (open romboids), polar ring S0s and spirals (crosses) and polar ring ellipticals (asterisks). Filled squares represent the reference values derived from the comparison sample of Bettoni et al. (2001b).

Sparke (1994) have studied in detail the dynamics of selfgravitating annuli of matter inclined to the principal axes of axisymmetric and triaxial potentials. If the strength of gas self-gravity is negligible, the inclined ring may rapidly settle towards the equatorial plane, appearing either as a co- or as a counter-rotating disk. In contrast, if the gas ring is heavy, the self-coupling can stabilise the ring for several Hubble times. In an intermediate case, several subrings (near polar or close to the equator), characterized by different precessing rates, can coexist in a single galaxy (e.g. NGC 660).

Studying the morphology of polar rings, van Gorkom et al. (1987) and Sage \& Galletta (1993) have found that their ages may vary from $400 \mathrm{Myr}$ to $\geq 4 \mathrm{Gyr}$, if the smooth rings are the oldest. Some polar rings or inclined rings could be the result of recent acquisitions, whereas others appear to be evolved systems. However, twisted polar rings are uncommon and some have had time to form stars. This requires the existence of a stabilising mechanism. The observations of atomic and molecular gas show that the quantity of gas mass present in polar rings is sufficient to stabilise them through self-gravity (van Gorkom et al. 1987; Sage \& Galletta 1993; Galletta et al. 1997, and this work). We derived a global content of cold gas $\left(M_{\text {gas }}\right)$ in polar rings which is 1-2 orders of magnitude higher than in normal galaxies.

In contrast to polar rings, the derived content of cold gas in counterrotators is close to normal. Although counterrotators and polar rings probably share a common 


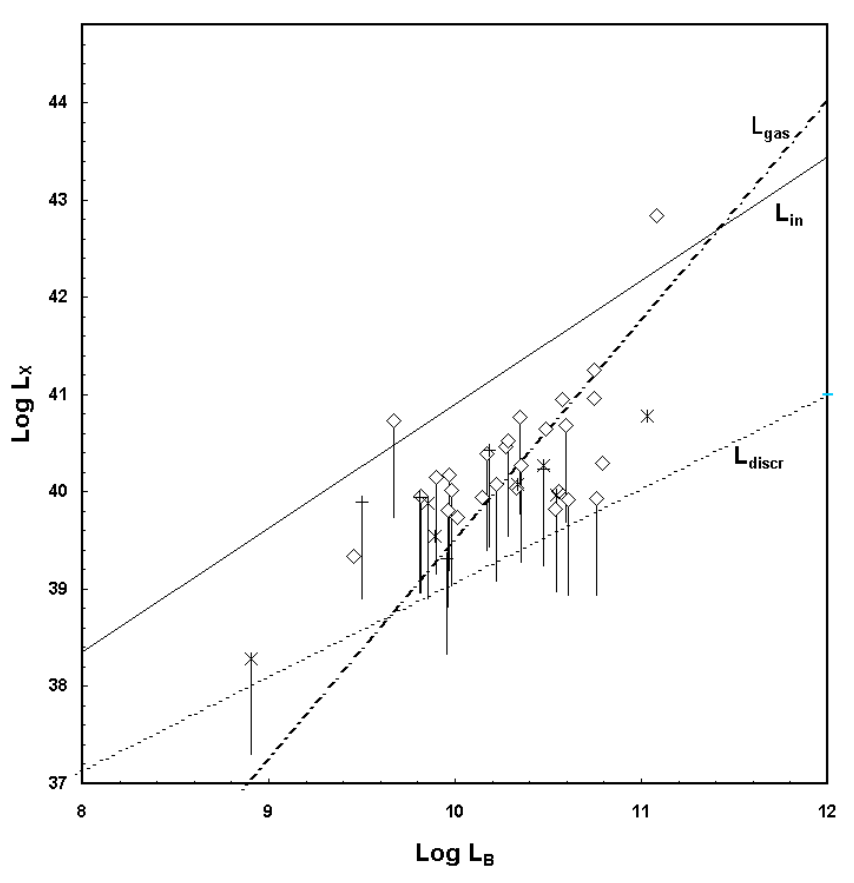

Fig. 8. Plot of $\log L_{\mathrm{X}}$ vs. $\log L_{B}$ for the accreting galaxies. Symbols are as in Fig. 3. The observed values are compared with different models including various X-ray luminosity sources: hot diffuse gas (dashed-dotted line labeled $L_{\text {gas }}$, discussed by Beuing et al. 1999), discrete sources (dashed line, labeled $L_{\text {discr }}$, discussed by Ciotti et al. 1991). All galaxies, except for NGC 4073, show luminosities lower than expected from steady-state cooling flow models, assuming the recent SN I rate 0.18 (Cappellaro et al. 1999).

origin, the estimated gas masses confirm that light gas rings may have evolved faster. If the mass of gas originally accreted is not sufficient to stabilise the ring through selfgravity, the ring settles toward the equatorial plane in less than a Hubble time. In this case, the merger relic could be a counterrotator. Once the gas disk has settled to the plane with an antiparallel spin it can interact with the gas of the primary disk. Since the two components have opposite rotating directions, there can be large-scale shocks and angular momentum annihilation when they come into contact. Near this transition region the transformation of atomic into molecular gas could be enhanced, especially if the primordial gas content is high, i.e., for late-type accretors. Confirming these expectations, the measured $M\left(\mathrm{H}_{2}\right) / M_{\mathrm{HI}}$ ratio seems larger in counterrotators than in normal galaxies for types $t>0$.

In the course of this process, a starburst might be triggered in the circumnuclear molecular gas disk (GarcíaBurillo et al. 2000). The time-scale for gas infall could be extremely short, being close to the free-fall time, i.e., $\sim 10^{7-8}$ Myr. The mass of gas involved in the starburst episode however is kept low enough $\left(10^{8-9} M_{\odot}\right)$ for a typical counterrotating galaxy. In polar rings, although the cold gas content is larger than the one in normal galaxies, star formation in the dynamically stable ring proceeds calmly. Confirming this scenario it was found that coun- terrotators, polar rings and normal galaxies have a similar content of hot gas, according to their normalized X-ray luminosities. Also, the normalized $L_{\mathrm{FIR}}$ lies within the typical boundaries of aged $(T \sim 1$ Gy) mild starbursts, far from the values characteristic of massive mergers (see Read \& Ponman 1998).

Acknowledgements. We would like to thank Dr. G. Paturel for kindly making available to the authors the FIR raw data of LEDA database and to Dr. F. Ochsenbein for the changes made to the Vizier's query form after our request. Thanks to the referee's comments for useful suggestions on the statistical analysis. This research made use of Vizier service (Ochsenbein et al. 2000) and of NASA's Astrophysics Data System Abstract Service, mirrored in CDS of Strasbourg. SGB and ARF thank financial support from the Spanish CICYT under grant number PB96-0104 and CICYT-PNIE under grant number 1FD19971442. GG has made use of funds from University of Padova (Fondi 60\%-2000).

\section{References}

Allam, S., Assendorp, R., Longo, G., Braun, M., \& Richter, G. 1996, A\&AS, 117, 39

Arnaboldi, M., \& Sparke, L. S. 1994, AJ, 107, 958

Arp, H. 1966, Pasadena: California Inst. Technology,

Arp, H. C., \& Madore, B. F. 1987, Catalogue of Southern Peculiar Galaxies and Associations (Cambridge Univ. Press), vol. 1 , p. 1

Bertola, F., \& Bettoni, D. 1988, ApJ, 329, 102

Bertola, F., \& Galletta, G. 1978, ApJ, 226, L115

Bertola, F., Galletta, G., Kotanyi, C., \& Zeilinger, W. W. 1988a, MNRAS, 234, 733

Bertola, F., Buson, L. M., \& Zeilinger, W. W. 1988b, Nature, 335,705

Bettoni, D. 1984, The Messenger, 37, 17

Bettoni, D., \& Galletta, G. 1997, A\&AS, 124, 61

Bettoni, D., Fasano, G., \& Galletta, G. 1990, AJ, 99, 1789

Bettoni, D., Galletta, G., \& Oosterloo, T. 1991, MNRAS, 248, 544

Bettoni, D., Della Valle, A., Marmo, C., \& Galletta, G. 2001a, in preparation

Bettoni, D., Galletta, G., \& García-Burillo, S. 2001b, in preparation (Paper II)

Beuing, J., Döbereiner, S., Böhringer, H., \& Bender, R. 1999, MNRAS, 302, 209

Braine, J., \& Combes, F. 1992, A\&A, 264, 433

Braun, R., Walterbros, R. A. M., \& Kennicutt, Jr., R. C. 1992, Nature, 360, 442

Bregman, J. N., Hogg, D. E., \& Roberts, M. S. 1992, ApJ, 387, 484

Burstein, D., Jones, C., Forman, W., Marston, A. P., \& Marzke, R. O. 1997, ApJS, 111, 163

Buta, R., van Driel, W., Braine, J., et al. 1995, ApJ, 450593

Caon, N., Macchetto, D., \& Pastoriza, M. 2000, ApJS, 127, 39

Cappellaro, E., Evans, R., \& Turatto, M. 1999, A\&A, 351, 459

Casoli, F., Dickey, J., Kazès, I., et al. 1996, A\&AS, 116, 193

Casoli, F., Sauty, S., Gerin, M., et al. 1998, A\&A, 331, 451

Ciotti, L., Pellegrini, S., Renzini, A., \& D'Ercole, A. 1991, ApJ, 376, 380 
Da Costa, L. N., Pellegrini, P. S., Davis, M., et al. 1991 ApJS, 75,935

Dettmar, R. J., Jullien-Dettmar, M., \& Barteldrees, A. 1990, in The Interstellar Medium in External Galaxies, ed. D. J. Hollenbach, \& H. A. Thronson, NASA CP-3084, 246

de Vaucouleurs, G., de Vaucouleurs, A., Corwin, H. G., et al. 1991, Third Reference Catalogue of Bright Galaxies (RC3), (Springer-Verlag: New York)

Ebneter, K., \& Balick, B. 1985, AJ, 90, 183

Forbes, D. A., Franx, M., \& Illingworth, G. D. 1994, ApJ, 428, L49

Fabbiano, G., Kim, D.-W., \& Trinchieri, G. 1992, ApJS, 80, 531

Fouque, P., Gourgoulhon, E., Chamaraux, P., \& Paturel, G. 1992, A\&AS, 93, 211

Franx, M., \& Illingworth, G. D. 1988, ApJ, 327, L55

Galletta, G. 1996, ASP Conf. Ser. 91, IAU Colloq. 157, Barred Galaxies, 429

Galletta, G., Sage, L. J., \& Sparke, L. S. 1997, MNRAS, 284, 773

García-Burillo, S., Sempere, M. J., \& Bettoni, D. 1998, ApJ, 502,235

García-Burillo, S., Sempere, M. J., Combes, F., Hunt, L. K., \& Neri, R. 2000, A\&A, 363, 869

Haynes, M. P., Jore, K. P., Barrett, E. A., Broeils, A. H., \& Murray, B. M. 2000, AJ, 120, 703

Harsoula, M., \& Voglis, N. 1998, A\&A, 335, 431

Hawarden, T. G., Longmore, A. J., Tritton, S. B., Elson, R. A. W., \& Corwin, H. G., Jr. 1981, MNRAS, 196, 747

Hernquist, L., \& Barnes, J. E. 1991, Nature, 354, 210

Huchtmeier, W. K. 1982, A\&A, 110, 121

Huchtmeier, W. K. 1997, A\&A, 319, 401

Kenney, J. D., \& Young, J. S. 1988, ApJS, 66, 261

Knapp, G. R., Guhathakurta, P., Kim, D.-W., \& Jura, M. 1989, ApJS, 70, 329

Kannappan, S. J., \& Fabricant, D. G. 2001, AJ, 121, 140

Magri, C. 1994, AJ, 108, 896

Malin, D. F 1985, New Aspects of Galaxy Photometry, ed. J.-L. Nieto (Berlin: Springer-Verlag), 27

Ochsenbein, F., Bauer, P., \& Marcout, J. 2000, A\&AS, 143, 23

Paturel, G., Andernach, H., Bottinelli, L., et al. 1997, A\&AS, 124,109

Quinn, T. \& Binney, J. 1992, MNRAS, 255, 729

Read, A. M., \& Ponman, T. J. 1998, MNRAS, 297, 143

Richter, O. G., Sackett, P. D., \& Sparke, L. S. 1994, AJ, 107, 99
Roberts, M., Hogg, D. E., Bregman, J. N., Forman, W. R., \& Jones, C. 1991, ApJS, 75, 751

Rubin, V. C. 1994, AJ, 108, 456

Rubin, V. C., Hunter, D. A., \& Ford, K. W. Jr. 1991, ApJS, 76,153

Rubin, V. C., Graham, J. A., \& Kenney, J. D. P. 1992, ApJ, 394, L9

Sage, L. J. 1993, A\&A, 272, 123

Sage, L. J., \& Galletta, G. 1993, ApJ, 419, 544

Sage, L. J., \& Galletta, G. 1994, AJ, 108, 1633

Schweizer, F., Whitmore, B. C., \& Rubin, V. C. 1983, AJ, 88, 909

Schweizer, F., van Gorkom, J. H., \& Seitzer, P. 1989, ApJ, 338 , 770

Sparke, L. S., 1986, MNRAS, 219, 657

Sparks, W. B., Wall, J. V., Thorne, D. J., et al. 1985, MNRAS, 217,87

Steiman-Cameron, T. Y., \& Durisen, R. H. 1982, ApJ, 263 L63

Strong, A. W., et al. 1988, A\&A, 207, 1

Thakar, A. R., \& Ryden, B. S. 1996, ApJ, 461, 55

van Driel, W., \& Buta, R. 1993, PASJ, 45, 47

van Driel, W., Arnaboldi, M., Combes, F., \& Sparke, L. S. 2000, A\&AS, 141, 385

van Gorkom, J. H., Schechter, P. L., \& Kristian, J. 1987, ApJ, 314,457

Veron-Cetty, M. P., \& Veron, P. 2000, ESO Scient. Rep., 19, 1

Voglis, N., Hiotelis, N. \& Höfflich, P. 1991, A\&A, 249, 5

Vorontsov-Velyaminov, B. A. 1959, Atlas and catalog of interacting galaxies

Young, J. S., \& Knezek, P. M. 1989, ApJ, 347, L55

Young, J. S. Xie Shuding, Tacconi, L., et al. 1995, ApJS, 98, 219

Walsh, D. E. P., van Gorkom, J. H., Bies, W. E., et al. 1990, ApJ, 352, 532

Wang, Z., Schweizer, F., \& Scoville, N. Z. 1992, ApJ, 396, 510

Watson, Dan, M., Guptill, Matthew, T., Buchholz, \& Leah, M. 1994, ApJ, 420, 21

Wiklind, T., \& Henkel, C. 1990, A\&A, 227, 394

Williams, B. A., \& van Gorkom, J. H. 1995, in Groups of Galaxies, ed. O. G. Richter, \& K. Borne, ASP Conf. Ser., 70,77

Whitmore, B. C., Lucas, R. A., McElroy, D. B., et al. 1990, AJ, 100, 1489

Zhu, M., Seaquist, E. R., Davoust, E. Frayer, D. T., \& Bushouse, H. A. 1999, AJ, 118, 145 